\author{
FORCES ON A HYDRAULIC VALVE SPOOL \\ Palumbo,A. ${ }^{* *}$, Paoluzzi,R. ${ }^{*}$, Borghi, M. $^{\$}$ Milani, $M .^{\sim}$ \\ * Cemoter C.N.R., Via Canal Bianco, 2844044 Cassana-Ferrara, ITALY \\ ** Former student of University of Modena at Cemoter \\ $\$$ University of Bologna, viale Risorgimento 2, Bologna, ITALY \\ Doctorate student, University of Modena, Via Campi 213/b 41100 Modena, ITALY
}

\begin{abstract}
The analysis and the evaluation of the steady-state flow forces acting on the spool of hydraulic valves is a keyaspect in performance assessment of many working conditions. These flow forces are generated by the different pressures on the spool faces caused by the high gradients of velocity near the metering orifice edge. In some working conditions these forces can cause significant instabilities in valve operation and a better insight is needed, with respect to classical approximations, in order to acquire informations on its causes and to guide design changes.

An experimental and numerical analysis has been carried out in order to analyse the overall force needed to operate a valve spool in different working conditions in a controlled environment.

From the experimental side, test results were used in order to identify critical operating conditions, and C.F.D. flow analysis (computational fluid dynamics based on the finite element method) was checked against the measured value of the operating force in order to tune a numerical model for a parametric investigation of valve flow characteristic. The paper focuses on the numerical aspect of the study, highlighting possibilities offered by C.F.D. in hydraulics, its expected degree of approximation, and problems in comparing numerical results with experimental evidence. Some detail considerations are reserved to a sensitivity analysis on aspects usually unknown in setting up a numerical C.F.D. analysis, such as turbulence model parameters and effect of geometric simplification.
\end{abstract}

Keywords: Flow forces, Computational Fluid Dynamics, Hydraulic valves

\section{General Background}

The spool of an hydraulic valve or distributor, is subjected to a set of forces (i.e. actuation, friction, inertia, pressure and flow) that can be decomposed into their axial and radial components. Radial components, if not properly balanced, are likely to cause contact between spool and land, thus inducing relevant friction forces (in the axial direction), which could be shown to be proportional to the static friction coefficient and the overall pressure difference across the clearance space. Axial forces on the spool are well known and documented in many basic textbooks, although their effective value remains to a large extent unknown in actual operation, major interest must be addressed to the flow forces, as their relative influence in some particular operating conditions may become extremely significant in determining the overall actuating force necessary to force spool movement.

The effect of flow forces on hydraulic valve spool was thoroughly studied [1], and, generally, simplified solutions based on the momentum equation are considered satisfactory to the purpose of flow force compensation in valve spools.

According to the analysis above, it can be shown that flow forces can be expressed as:

$$
F_{f}=2 \cdot C_{d} \cdot C_{e} \cdot \pi D \cdot \Delta p \cdot \cos \vartheta \cdot x
$$

where $C_{\mathrm{d}}$ and $C_{\mathrm{e}}$ are discharge coefficients and other symbols have the meaning shown in FIGURE 1.

In the simplifying assumption that the pressure droop remains constant with the variation of $\mathrm{x}$ (which could happen for instance in controlled pressure systems) the relationship becomes linear and various spools can be compared simply by evaluation of the rate $2 \cdot C_{d} \cdot C_{c} \cdot \pi D \cdot \Delta p$. This relation holds up to the flow saturation conditions, where a quadratic decrease of the flow force with $\mathrm{x}$ starts [2].

Flow force compensation action can start from this analysis, reshaping the spool section in order to obtain flow force in the form:

$$
F_{f}=\rho \cdot v \cdot Q \cdot\left(\cos \vartheta_{1}-\cos \vartheta_{2}\right)
$$

where the symbols have the meaning shown in 


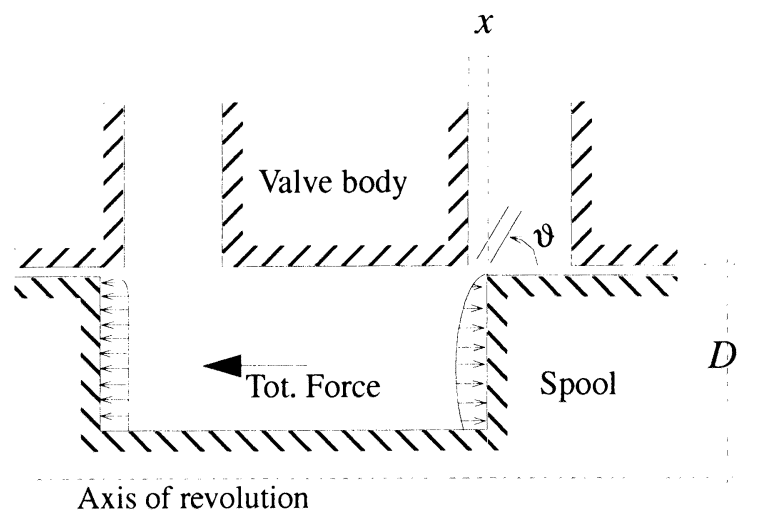

FIGURE 1 Generic sketch of flow force on spool FIGURE 2.

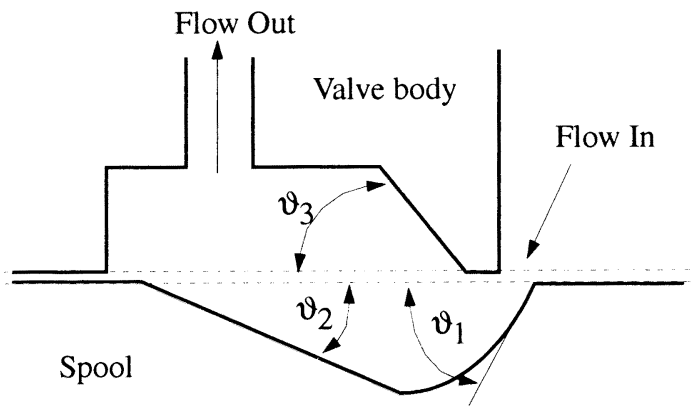

FIGURE 2 Generic flow force compensation

Properly shaped spools can therefore make the flow force positive, negative or approximately null, according to the value of the angles $\vartheta_{1}$ and $\vartheta_{2}$.

This generally accepted theory was subjected to criticism by some experimental evidences shown in [3], where some weak points of the Von Mises assumptions were pointed out as possible source of misleading results, both for uncompensated $(\vartheta=90 \mathrm{deg})$ and compensated spools.

\section{The AD5 valve}

AD5 is a spool type $4 / 3$ distributor with a rated/ maximum flow 75/100 1/min. and maximum working pressure $320 \mathrm{bar}$, is spring-centred and driven by two solenoids (DC or $\mathrm{AC}$ ) with $3 \mathrm{~Hz}$ maximum excitation frequency.

A cross-sectional view of the valve is shown in FIGURE 3. In the configuration tested, the valve has a closed centre position with positive overlap.

\section{Numerical model}

In order to carry out simulation studies on the valve under investigation, the solution of the NavierStokes equations describing the flow must be

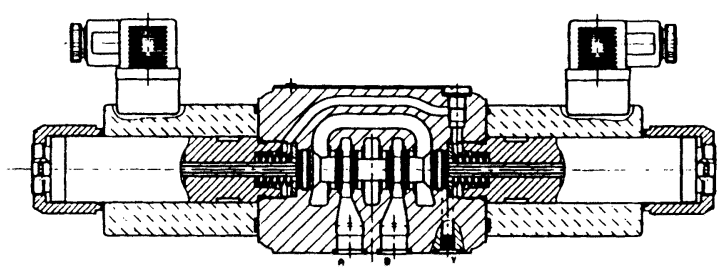

FIGURE 3 Cross-sectional view of Aron AD5 valve

obtained by means of techniques able to describe adequately the domain. Among the available techniques, a discretization based on the Finite Element approach was used. Two commercial software packages were used to the purpose of calculation, Flotran 2.1 by Compuflo Inc. [5] and FIDAP 7.07 by Fluid Dynamics International [6], both of them featuring laminar and turbulent internal and external flows.

As a full 3-D analysis can hardly be fitted on a standard workstation, a forced choice was to limit the analysis to a $2 \mathrm{D}$ or axisymmetric geometry.

\subsection{Geometry}

The simplification of the actual geometry into a 2-D axisymmetric model imposes the choice of a reference system centred on the spool axis. Because of the symmetry of the flow metering edges, the only portion of the model represented is the passage from $\mathrm{P}$ to A ports, disregarding completely the B-T connection. A further simplification is less immediate and refers to the inflow and outflow sections chosen. These refer to a non-axisymmetric portion of the model, and, in the general case, would require a more detailed description, both from the geometric and boundary conditions point of view; however, considerations related to the dimension of the problem under investigation, suggested to limit the model to a clear-cut of the inlet and outlet sections of the valve, assuming that the flow distortion thus induced could be considered negligible to the purpose of the global result on the pressure field on the spool surface.

The resulting geometry can be desumed by the mesh plot shown in FIGURE 4. The total number of elements and their order is not relevant at this stage and will be discussed later.

\subsection{Turbulence model}

Turbulence effects are usually considered in the formulation of the Navier-Stokes as a fluctuating stochastic quantity superimposed to averaged quantity describing the mean flow [7]. The new unknowns 
thus introduced in the system of equations require new equations to be introduced in the formulation, these usually come from some models of turbulence behaviour and are referred to as 'closure equations'. Among the possible models, both codes include the widely used $k$ - $\varepsilon$ model, where semi-empirical diffusivity equations are used for both the turbulence kinetic energy $k$ and the turbulence dissipation $\varepsilon$.

In spite of the relative simplicity of the model, a difficult task is to apply correct boundary conditions to the $k-\varepsilon$ field. As generally experimental values are not available, a possible profile to be used in the inlet section is given by

$$
\begin{aligned}
& k=\sqrt{c_{\mu}} \cdot\left(l_{m} \frac{d u}{d n}\right) \\
& \varepsilon=\frac{c_{\mu} k^{2}}{l_{m}^{2} \cdot\left|\frac{d u}{d n}\right|}
\end{aligned}
$$

where $c_{\mu}$ is an empirical constant whose value is assumed to be 0.009 and comes from the useful definition of the turbulent viscosity $\mu_{\mathrm{t}}$ as

$$
\mu_{t}=c_{\mu} \rho \frac{k^{2}}{\varepsilon}
$$

The definitions given in equations 3 and 4 allow the computation of boundary values for $\mathrm{k}$ and $\varepsilon$, provided that the velocity profile is known as a function of the distance from the inlet section axis, and the mixing length $\mathrm{l}_{\mathrm{m}}$ is estimated. To this purpose some approximate relations can be used as a function of the local Reynolds number of the fluid.

From this point on, the two approaches used by FLOTRAN and FIDAP differ. In fact FIDAP allows a direct definition of both $\mathrm{k}$ and $\varepsilon$ profiles according to the defined laws; conversely FLOTRAN requires the turbulent kinetic energy at the inlet section defined as a fraction of the kinetic energy of the mean flow according to

$$
k=\frac{3}{2}(I \cdot U)^{2}
$$

where $\mathrm{I}$ is the inlet intensity factor of turbulent kinetic energy and $U$ is the nodal value of the averaged flow. Typical values are generally in the range $0.01-0.1$. Consequently values for the inlet dissipation factor $\left(\mathrm{I}_{\mathrm{S}}\right)$ can be defined according to:

$$
\varepsilon=\frac{c_{\mu} k^{\frac{3}{2}}}{I_{s} L}
$$

where $\mathrm{L}$ is the characteristic length scale of the problem.

These preliminary considerations allow a first comparison between the two codes, in fact FIDAP features a detailed control of the boundary conditions on the $k-\varepsilon$ field, whilst FLOTRAN requires estimation of the relative importance of the turbulence with respect to the mean flow. However, in both cases reasonably good values can be estimated for both approaches.

\subsection{Solution techniques}

Before any attempt of solution to the problem, its specific character from the numerical point of view must be investigated. This require consideration on both expected values of the computed quantities and solution algorithms to be applied.

\subsubsection{Nondimensionalization}

Typical dimensions of the metering passage of the valve under investigation are of the order of magnitude of a fraction of millimetre $\left(10^{-4} \mathrm{~m}\right)$, while values for pressure are typically in the range of $10^{7} \mathrm{~Pa}$ and for speed $10^{2} \mathrm{~m} / \mathrm{s}$. This means that when the problem is described in SI units, variables values can span over 11 orders of magnitude, and this can cause serious numerical problems. The immediate consequence is that, in order to allow good performance of the algorithms, the problem must be entirely non dimensionalized. This is not a difficult task for laminar problems, but, under some circumstances, it can become more complex and non-unique; moreover, when the $k-\varepsilon$ model equations are used, two additional equations must be non-dimensionalized.

The additional work needed in these cases is paid in terms of increased model generality and solution procedure stability.

\subsubsection{Solution strategy}

In the two software packages used, the solution strategy differs considerably. This of course should not affect the final solution, but can affect the convergence rate and even the possibility of obtaining a converged solution. A detailed analysis of the two solution strategies adopted is beyond the scope of this paper, but it is worth noting that FIDAP allows 
a much higher control over the solution strategy and on the parameters of the solver, thus allowing a more efficient convergence rate of the solution to be achieved. The solution strategy was a semi-direct pressure integration for Flotran and a Successive Substitution scheme for FIDAP.

\section{Numerical results}

The final model obtained is shown in FIGURE 4. It

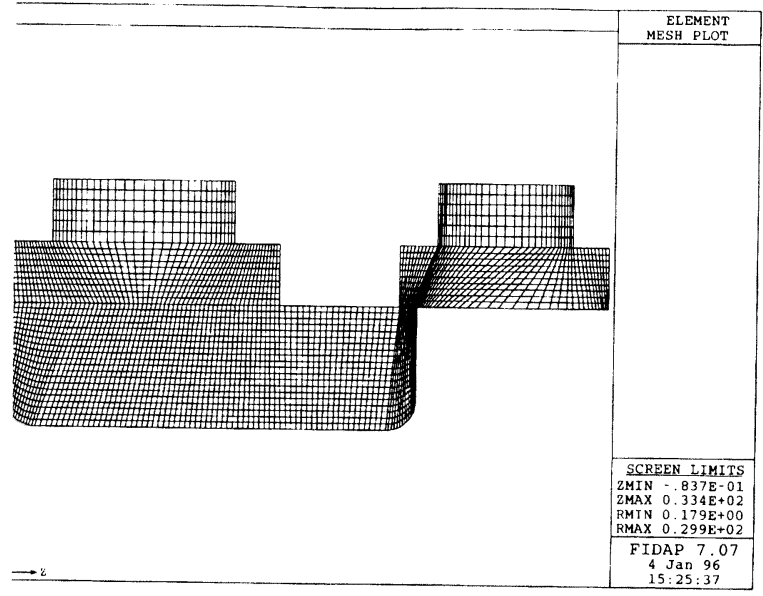

FIGURE 4 Mesh of the model (both codes)

is a snapshot of the spool position when the dimension of the metering edge is $0.6 \mathrm{~mm}$. It was decided, as a first investigation, to use the same mesh for both codes, even if this approach is not completely correct as it does not take advantage of the particular element formulations available in FIDAP, but was anyhow considered a first step for solution comparison.

The model used 3808 four-node quadrilateral linear elements (a total of 3625 nodal points).

The boundary conditions applied in both codes were the same (a pressure differential of 146 bar, no slip conditions at fluid-wall interface and free flow conditions at the outlet port) and the problem was nondimensionalized with respect to the metering section.

The expected maximum value of the velocity (used also as reference value for speed and pressure nondimensionalization) is $182.7 \mathrm{~m} / \mathrm{s}$, leading to a Reynolds number of 1910 , while the value computed in the inlet section is $2.8 \mathrm{~m} / \mathrm{s}$, with a correspondent value of the Reynolds number of the flow of 293.

A first interesting result is that no significant variation was noticed in the solution varying the values of the $\mathrm{k}-\varepsilon$ parameters from those computed from the metering section, to inlet section or to program defaults. Only the convergence rate was affected (or even hindered); best values were achieved with values computed for the actual section (inlet).

As far as flow forces are concerned, the main effect is due to the pressure distribution on the vertical face of the spool upstream the metering edge. To the purpose of force computation, pressure values must be integrated over the complete spool surface taking into account the axisymmetric design, however, for comparison purposes, considering that the geometry is the same and that the pressure drop and flow acceleration occur in a confined region close to the metering edge, this last pressure distribution only can be considered.

A contrast of distribution computed with Flotran and FIDAP is shown in FIGURE 5. It can be shown

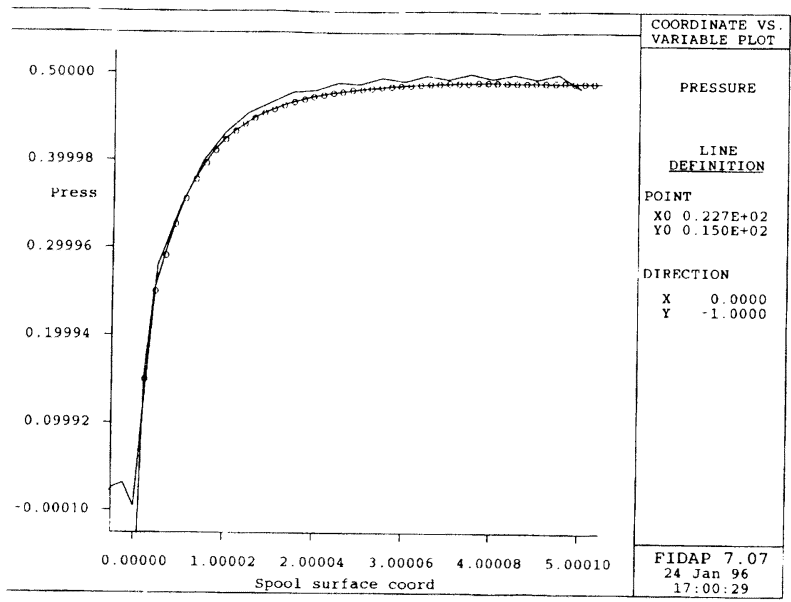

FIGURE 5 Pressure distribution along the vertical spool surface (Flotran values are marked by "o")

that the two codes give the same result with a remarkable precision, although the computational time required by FIDAP was considerably lower (even taking into account that the two programs run on different workstations).

Also other general representations of the flow are almost identical with the two codes, therefore only results available from FIDAP are shown in the following. For instance, FIGURE 6 shows the contoured values of the velocity module, showing that the overall values of the computed velocity are slightly lower than those estimated by approximated formulas (an adimensional value of 1 means perfect identity) and that, as a general remark, the shape of the outflow land towards A port, causes the flow to be deflected further, causing a local increase of the pressure values (FIGURE 7) and a subsequent energy dissipation (FIGURE 8).

The total force on the spool, computed with both programs in this particular configuration, is $258.7 \mathrm{~N}$ (in the direction of valve closure), a value which 


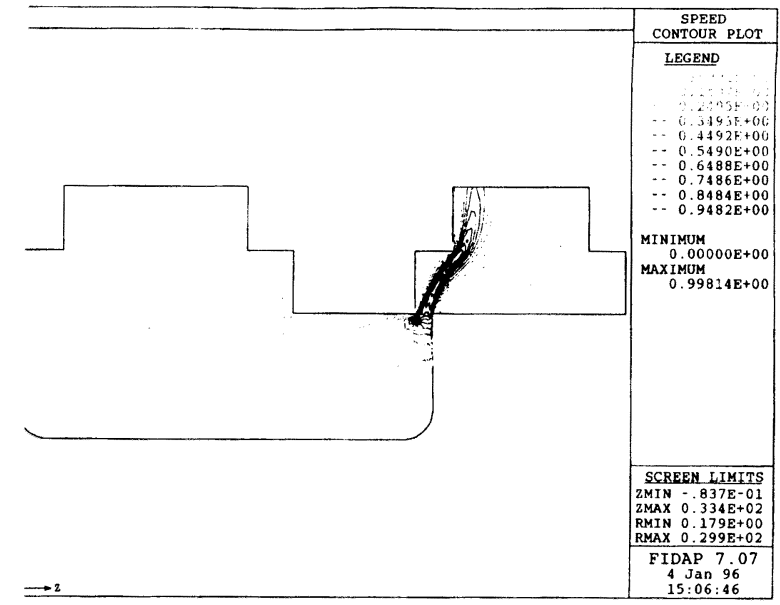

FIGURE 6 Speed contour on the valve

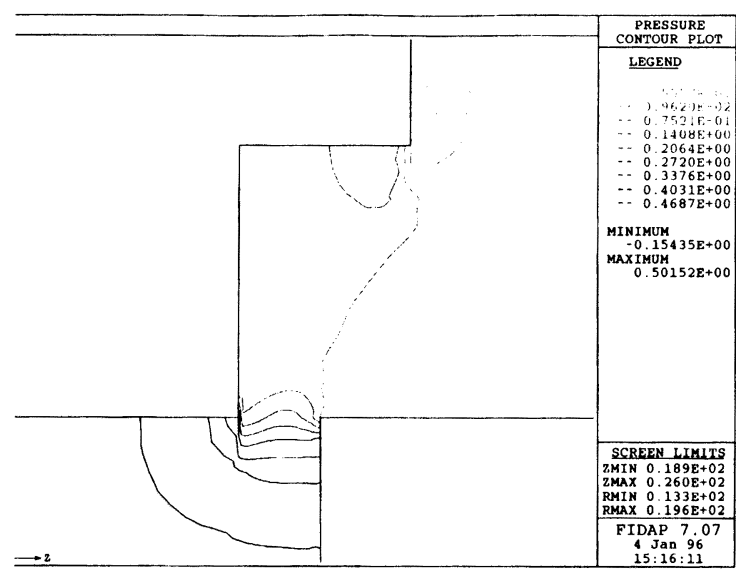

FIGURE 7 Close-up of pressure distribution in the valve

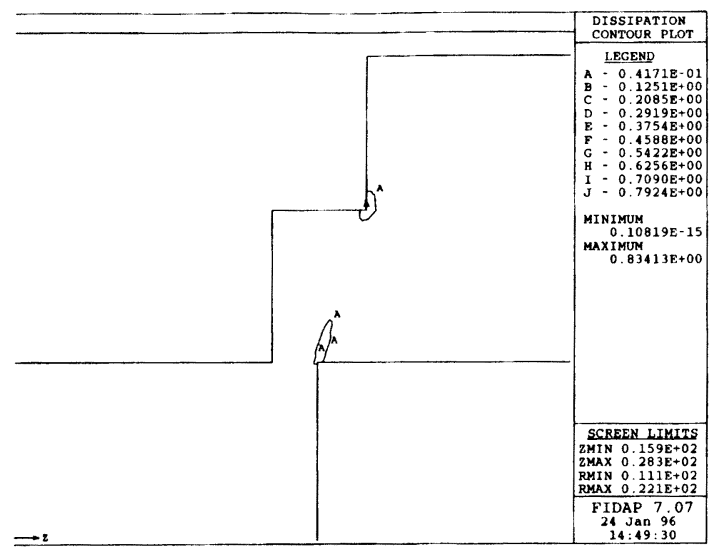

FIGURE 8 Energy dissipation ( $\varepsilon$ field)

was proved to be completely insensitive to variations in the values of the $k$-e model parameters [2]. The corresponding value of the theoretical flow force computed from Equation 2 is $212.5 \mathrm{~N}$ (18\% lower than CFD value). The reasons of the observed difference is of course partly due to the different approximations implied by the two computations, and only experimental measurements can help in deciding which one is the most reliable approximation of actual valve behaviour.

\section{Experimental Setup}

The evaluation of the flow forces starting from the developed pressure field on the spool surface is a formidable task. It is a typical example of a case where measurements can modify the physical phenomenon. Therefore, flow forces effects can be measured, rather than their causes. To this purpose a special valve was designed, in order to be able to measure the overall forces acting on the valve spool at fixed positions, under controlled flow rate or pressure differential.

\subsection{Test Rig}

The test rig was at the first stage set up by the valve manufacturer according to the schematic shown in FIGURE 9. An electrical motor drives a variable

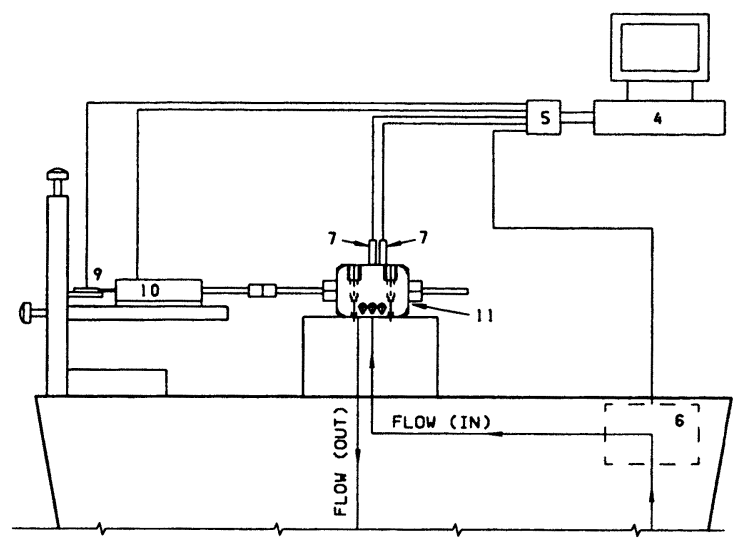

FIGURE 9 Schematic of the test rig

displacement pump feeding a supply circuit fitted with a pressure relief valve. The Ad5 valve in its closed centre configuration is inserted in the circuit and the flow rate and pressure values at the four ways of the valve are recorded by transducers.

The valve is designed in order to have the possibility of measuring both spool position and force by means of dedicated transducers.

Data acquisition is performed by a 486DX2 Personal Computer, with A/D board and dedicated data acquisition software.

Tests are planned in different valve operating conditions, both in terms of spool position, flow rate and pressure differential. Port connections are being tested with both P-A and B-T flow, to simulate 
actual operating conditions, and with P-A flow only (B and T ports connected to tank) in order to acquire data suitable for comparison with the computation.

\subsection{Experimental Results}

Experimental results are presently being collected and only preliminary results (mainly functionality tests) are available at this moment.

Preliminary tests, FIGURE 10, already evidenced

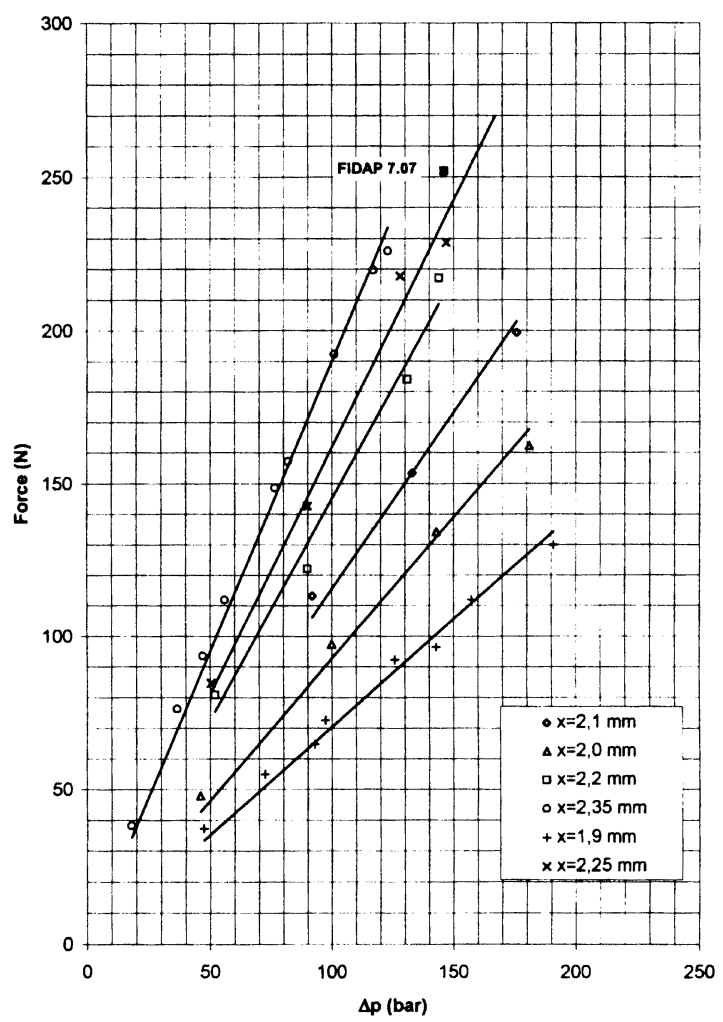

FIGURE 10 Total spool force as a function of $\Delta p$

the need for an extremely accurate evaluation of undesired influences of valve unknown parameters which can affect the measure, such as spool-shaft concentricity, spool sticking, seal conditions and, eventually, effect of the internal leakage and trapped fluid volumes. A finest comparison between CFD and tests data show a good agreement (experimental and numerical values show have the same regression curve), and both are slightly higher than the corresponding value given by Equation 1 .

\section{Concluding Remarks}

An approach to the evaluation of the static flow forces on the valve spool was presented mainly from the computational point of view, taking advantage of the use of two different commercial CFD codes, allowing some general comments to be drawn up:

- fluid power problems generally require a turbulent model to be used and the $\mathrm{k}-\varepsilon$ model generally perform satisfactorily;

- the $\mathrm{k}-\varepsilon$ model parameters are not critical to the purpose of 'high-level' quantity computation. Only convergence rate and detailed microscopic values are affected;

- different codes generally give the same overall results, although difference exist, mainly due to specific element descriptions, in local values of both pressure and velocity field;

- overall flow force values obtained are comparable with those computed by CFD and slightly higher than those computed by traditional engineering approximations; but, due to the simplifications induced by both methods, only a comprehensive experimental evidence can give a definite answer on the effectiveness of CFD methods to this purpose.

An extensive test activity is presently being starting to the purpose of data collection, and the test stand arrangement was shown together with some preliminary results.

\section{Acknowledgements}

This activity was partly carried out as Master of Science Thesis dissertation by the University of Modena, with an effective and strong cooperation of Aron SrL. The authors are grateful to all the Aron $\mathrm{R} \& \mathrm{D}$ staff for support in preparation of the AD5 special test valve and first test rig.

\section{References}

1. Blackburn, J.F., Reethof, G., Lowen Sharer, J., Fluid Power Control, John Wiley \& Sons, 1960

2. Palumbo, A., Analisi teorico-sperimentale delle forze di flusso in un distributore a cassetto a comando elettrico, MSc Thesis, University of Modena, 1995 (in italian)

3. Lugowsky, J., Experimental investigation on the origin of flow forces in hydraulic piston valves, 10th International Conference on Fluid Power, BHR, Brugges, B, 5-7 Apr. 1993.

4. Ferretti, G., Paoluzzi, R., Zarotti, L.G., CFD flow analysis of steady-state flow forces on valves, Procs. of the 2nd ISFP Sganghai, China,1995

5. Flotran 2.1 User's Manual, COMPUFLO, CHarlottesville, USA.

6. FIDAP 7.07 User's Manual, Fluid Dynamics International, Evanston, IL, USA, 1993 